\title{
Chiral Restoration in the Nuclear Medium
}

\section{$\mathrm{C}_{\text {Djalali }}{ }^{1}$, R Nasseripour ${ }^{1}$, D P Weygand ${ }^{2}, \mathrm{M} \mathrm{H} \mathrm{Wood}^{3}$ and CLAS Collaboration}

\author{
${ }^{1}$ University of South Carolina, Columbia, SC 29208 \\ ${ }^{2}$ Thomas Jefferson Accelerator Facility, Newport News, VA 23606 \\ ${ }^{3}$ University of Massachusetts, Amherst, MA 01003
}

\begin{abstract}
The photoproduction of vector mesons on various nuclei has been studied using the CLAS detector at Jefferson Laboratory. The vector mesons, $\rho, \omega$, and $\rho$, are observed via their decay to $e^{+} e^{-}$, in order to reduce the effects of final state interactions in the nucleus. Of particular interest are possible in-medium effects on the properties of the $\rho$ meson. The $\rho$ mass spectrum is extracted from the data on various nuclei, $\mathrm{D}_{2}, \mathrm{C}, \mathrm{Fe}$, and $\mathrm{Ti}$. We observe no significant mass shift and some broadening consistent with expected collisional broadening for the $\rho$ meson.
\end{abstract}

Keywords: Medium modifications, vector mesons, di-lepton decay.

PACS: 25.20.Lj, 13.20.-v, 13.60.Le, 14.40.-n.

\section{INTRODUCTION}

The theory of the strong interaction, Quantum Chromodynamics (QCD), has been extremely successful in describing high-energy and short-distance-scale experiments involving quarks and gluons. Applying QCD to low energy and large-distance-scale experiments has however been a major challenge. Although the rapidly increasing strength of the interaction in this latter case makes it impossible to apply perturbative techniques, the symmetries of QCD (such as chiral symmetry) provide guiding principles to deal with strong interaction phenomena. Various QCD-inspired predictions are now available in the non-perturbative domain, which can be tested experimentally at current hadron and electromagnetic facilities.

An interesting and puzzling problem of hadronic physics is the question of the origin of the large hadron masses $\left(\sim 1 \mathrm{GeV} / \mathrm{c}^{2}\right)$, which are typically two orders of magnitude larger than the masses of the light current quarks composing them $(<10$ $\mathrm{MeV}$ ). Almost four decades ago, the suggestion was made that much of the hadron mass is generated dynamically. Hadron masses are somewhat effected by the spontaneous breaking of chiral symmetry. One of these QCD "inspired" predictions is that in hot (finite temperature) and/or dense (finite density) matter, a chiral phase transition takes place and the broken chiral symmetry is restored resulting in a modification of the properties of hadrons (vector mesons in particular) in nuclear matter from their free-space values. This prediction has generated much interest, and there is an urgent need for experimental data to confirm or refute these predictions in this as yet largely unexplored domain.

CP947, VII Latin American Symposium on Nuclear Physics and Applications

edited by R. Alarcon, P. L. Cole, C. Djalali, and F. Umeres

(C) 2007 American Institute of Physics 978-0-7354-0461-8/07/\$23.00 
At high temperature or pressure, chiral symmetry may likely be restored. At normal nuclear densities, partial restoration of chiral symmetry may affect the properties of hadrons, in particular masses and widths. ${ }^{[1-6]}$

The first possible indication of a medium-affected $\rho$ meson mass came from the CERES ${ }^{7}$ and HELIOS $/ 3^{8}$ collaborations at CERN in 1995. The CERES collaboration reported on measurements of low-mass $\mathrm{e}^{+} \mathrm{e}^{-}$pairs from $\mathrm{p}-\mathrm{Au}$ and $\mathrm{Pb}-\mathrm{Au}$ collisions. Dilepton spectroscopy allows measurement of the in-medium properties without distortion due to final state interactions (FSI). While their proton-induced data could satisfactorily be accounted for by summing various hadron decay contributions, an enhancement over the hadronic contributions was observed for the $\mathrm{Pb}-\mathrm{Au}$ data in the mass range between 300 and $700(\mathrm{MeV} / \mathrm{c} 2)$. The same year, theorists were able to account for the observations by using a relativistic transport model assuming a decrease in the mass of the $\rho$ meson ${ }^{9}$. It has been suggested that "traditional" effects, such as an in-medium modified pion dispersion relation may be able to provide enough enhancement.

A better understanding of hadron properties in a hot and/or dense environment is therefore one of the most important endeavors of hadronic physics today. The change

of properties of the vector mesons in the medium is a hot topic that is currently being studied at RHIC (BNL) and HADES (GSI) and will be studied at ALICE (CERN) by measuring low mass di-lepton production. Although vector mesons preferentially decay into pions, the large final state interactions of the pions with the nuclear medium makes it almost impossible to derive any direct information about the meson properties in the medium. In a heavy-ion collision the final di-lepton yield is obtained by integrating over different densities and temperatures, and discriminating between different scenarios of the in-medium modifications for the vector mesons is difficult.

In their initial stages, relativistic heavy-ion reactions originate far from equilibrium and the temperature and density evolve over time. However, all theoretical predictions of in-medium properties of vector mesons in photon or pion induced reactions allow one to study the hadron properties in an environment that is much closer to equilibrium (normal nuclear density and zero temperature). The predicted in-medium effects for the vector mesons by the different models are so large that they should have observable consequences already at normal nuclear density. An experiment like g7 is ideal to measure the photoproduction of vector mesons off nuclei and will give complementary information about the in-medium properties of mesons.

\section{THEORETICAL MODELS.}

Due to chiral symmetry restoration, the mass and width of vector mesons, such as $\omega, \rho$, and $\varphi$, are predicted to change with increasing density. Brown and Rho ${ }^{3}(\mathrm{BR})$, starting from an effective Lagrangian approach at low energy and zero density, suggest the same Lagrangian at high density, but with the masses and coupling constants that are modified according to the symmetry constraints of QCD. They proposed an in-medium scaling law that predicts a decrease in the mass of the vector meson by about $20 \%$ in normal nuclear matter. 
Hatsuda and Lee ${ }^{4}(\mathrm{HL})$, based on QCD sum rule calculations, obtained the spectral changes of the vector mesons in the nuclear medium. Their calculations result in a linear decrease of the masses as a function of density $\rho$ :

$$
\frac{m_{V M}(\rho)}{m_{V M}(\rho=0)}=1-\alpha \frac{\rho}{\rho_{0}}, \alpha=0.16 \pm 0.06
$$

While QCD based models predict a large downward shift of the vector meson masses, more "conventional" processes such as in-medium re-scattering predict no change in the vector-meson mass but a substantial increase in the width of the meson. These effects are density dependent, and, if present, should be observed at normal nuclear densities. Consequently, one should be able to observe the medium modifications of the properties of the hadrons in pion-, proton- or photon-induced reactions. Models based on nuclear many body effects predict a broadening in the width of the $\rho$ meson with increasing density. This prediction is based on the assumption that many body excitations may be present with the same quantum numbers and can be mixed with the hadronic states. ${ }^{5,6}$

\section{EXISTING DATA.}

An observation of a medium-modified $\rho$ meson mass has also been claimed by the KEK-PS collaboration in an experiment where $12 \mathrm{GeV}$ protons were sent on $\mathrm{C}$ and $\mathrm{Cu}$ targets and the $\mathrm{e}^{+} \mathrm{e}^{-}$pairs were detected ${ }^{10}$. However, the clearest evidence is obtained for the $\phi$ meson. By selecting low momentum $\phi$ mesons, the $\phi$ meson peak in the invariant mass spectrum develops a shoulder due to the possibly modified $\phi$ 's decaying in the nucleus. Since few $\phi$ 's decay inside the nucleus, one needs sufficient statistics after the momentum cuts to observe the shoulder.

Very recently, the Crystal Barrel/TAPS collaboration has reported a downward shift in the mass of the $\omega$, where the analysis focused on the $\pi^{0} \gamma$ decay of lowmomentum $\omega$ mesons photoproduced on a nuclear target ${ }^{11}$. The $\omega \rightarrow \pi^{0} \gamma$ channel is a very "clean" channel for studying $\omega$ mesons since the branching ratio for $\rho \rightarrow \pi^{0} \gamma$ is two orders of magnitude smaller. Data were taken for two nuclear targets $\left(\mathrm{H}_{2}\right.$ and $\left.\mathrm{Nb}\right)$ and compared after subtracting a huge background. An enhancement was found toward lower masses for $\omega$ mesons produced on the $\mathrm{Nb}$ target. One concern about this experiment is the $\pi^{0}$ re-scattering in the nuclear medium, which could distort the invariant mass spectrum.

Recently, the NA60 experiment at CERN SPS has studied low-mass muon pairs in $158 \mathrm{~A}-\mathrm{GeV}$ In-In collisions (see these proceedings). A strong excess of pairs is observed above the expectation from the neutral meson decays. The high statistics and good mass resolution of about $2 \%$ have allowed isolating the excess by subtraction of the known sources. The comparison of the $\rho$ spectral function with the prediction of the Rapp/Wambach for broadening, and that of Brown/Rho for the shift in the mass of the $\rho$ shows no shift in mass but a broadening in the width of the $\rho$.

The latest medium modification results reported in relativistic heavy ion experiments are not consistent with the KEK and TAPS conclusions. Experiments, 
such as g7 at JLab, that look for medium modifications in normal nuclear density at equilibrium are needed to disentangle the different mechanisms. All these experiments are yielding results complementary to each other and offer excitement, but no clear consensus has yet emerged between the various analyses and the question of the density-induced modifications on the properties of vector mesons is still wide open.

\section{THE CLAS EXPERIMENT.}

The data for this study were taken in 2002 using the CEBAF accelerator and the CLAS detector located in the Hall-B of the Jefferson Laboratory. A comprehensive description of CEBAF, the Continuous Electron Beam Accelerator Facility, can be found in the reference ${ }^{12}$, while reference ${ }^{13}$ gives an elaborate account on the CLAS, the CEBAF Large Acceptance Spectrometer, and other Hall-B equipments. CLAS is a nearly $4 p$-detector which was designed to track charged particles with momenta greater than $200 \mathrm{MeV} / \mathrm{c}$ over the polar angle range from $8^{\circ}$ to $142^{\circ}$, while covering up to $80 \%$ of the azimuth. The detector is made of 3 regions of drift chambers, time-offlight scintillators, Cerenkov counters (CC) and electromagnetic calorimeters (EC). The $\mathrm{e}^{+} \mathrm{e}^{-}$event selection and the rejection of the very large $\pi^{+} \pi^{-}$background were done through cuts on the $\mathrm{EC}$ and the $\mathrm{CC}$.

The target contained a liquid deuterium (LD2) cell and seven solid foils, each with a $1.2 \mathrm{~cm}$ diameter. The separation between targets was $2.5 \mathrm{~cm}$; the target nucleus was determined by the position of the production vertex.
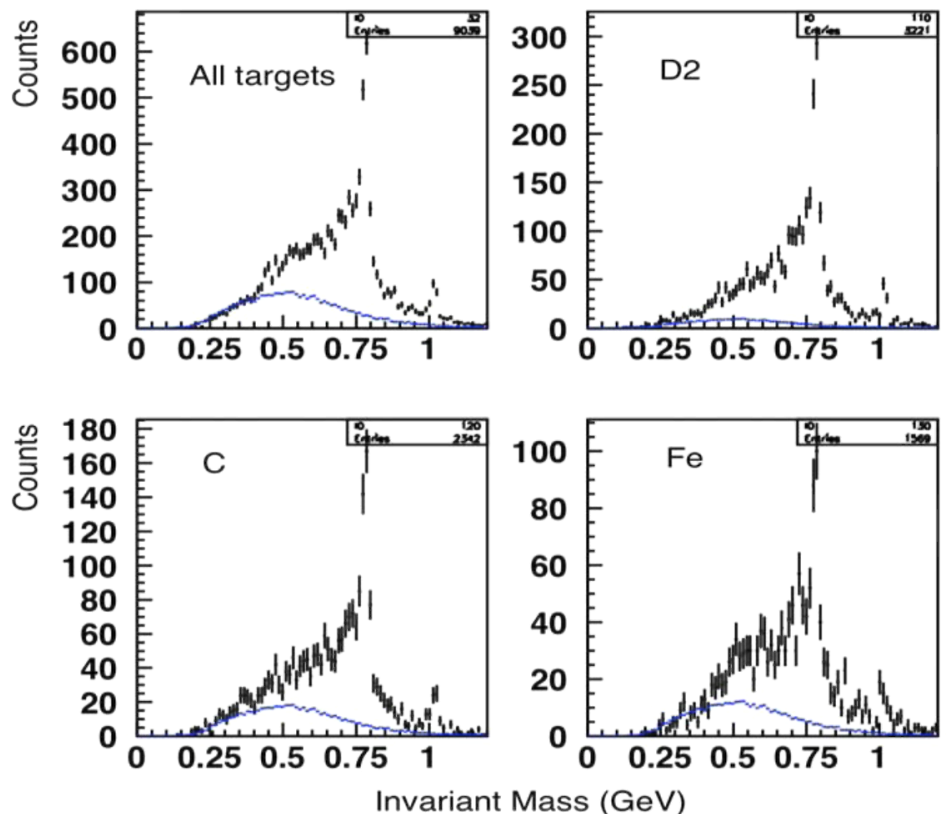

FIGURE 1. Normalized combinatorial background (blue) for all and individual targets compared to data (black). 
Lepton pair production has a background of random combinations of pairs due to the uncorrelated sources. The most salient feature of the uncorrelated sources is that they produce the same-charge lepton pairs as well as oppositely charged pairs. The same-charge pairs $\left(\mathrm{e}^{+} \mathrm{e}^{+}\right.$and $\left.\mathrm{e}^{-} \mathrm{e}^{-}\right)$provide a natural normalization of the uncorrelated background. This combinatorial method has successfully been used in the past for measurements involving opposite-sign pairs of pions or muons. ${ }^{14,15}$

The combinatorial background is statistically approximated by an event-mixing technique. The electrons of a given event are combined with positrons of another event, as the two samples of electrons and positrons are completely uncorrelated. This produces the phase-space distribution where electrons and positrons are actually from different processes but lying in the same event.

The mixed opposite-charged lepton chosen from samples of uncorrelated events was used to estimate the shape of the combinatorial background.

The result is shown in figure 1 for the individual targets as well as the total data.

\section{RESULTS AND DISCUSSION.}

To simulate each physics process, a realistic model was employed and corrected for the CLAS acceptance. The events were generated using a code based on a semiclassical Boltzmann-Uehling-Uhlenbeck (BUU) transport model developed by the group of $U$. Mosel at the University of Giessen which treats the photon-nucleus reactions as a two-step process. ${ }^{16}$ In the first step, the incoming photons react with a single nucleon taking into account the effects of shadowing.

Then in the second step, the produced particles are propagated explicitly through the nucleus allowing for final-state interactions, governed by the semi-classical BUU transport equations. A rather complete treatment of the $\mathrm{e}^{+} \mathrm{e}^{-}$pair production from $\gamma \mathrm{A}$ reactions at Jefferson Lab energies has been carried out using this code. ${ }^{17}$

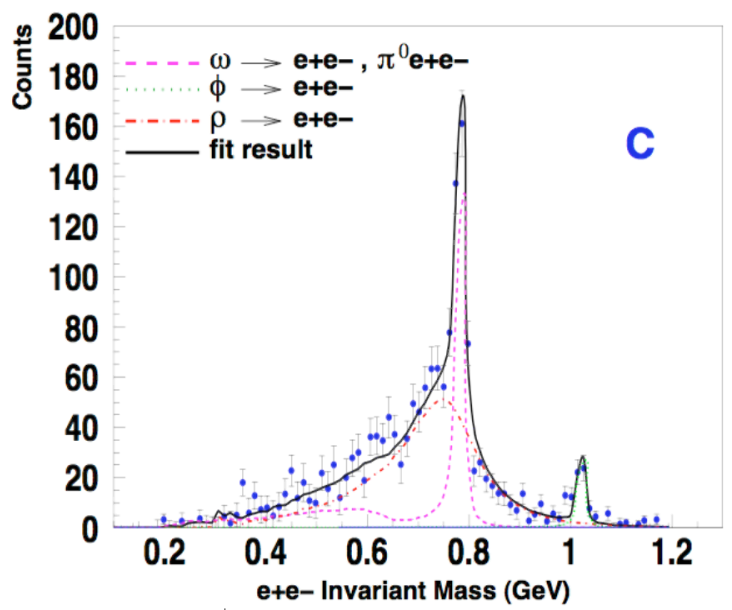

FIGURE 2. Results of the fit to the $\mathrm{e}^{+} \mathrm{e}^{-}$invariant mass obtained for $\mathrm{C}$ data. Curves are Monte-Carlo calculations by the BUU model ${ }^{18,19}$ for various $\mathrm{e}^{+} \mathrm{e}^{-}$channels. 
The expected combinatorial background distributions are subtracted from the $\mathrm{e}^{+} \mathrm{e}^{-}$ effective mass distributions. The shape of the narrow $\omega$ and $\phi$ vector mesons, and the $\omega$ Dalitz channel are well described by BUU model, and these distributions were fit to the data, then also subtracted, leaving just the experimental spectra of the $\rho$ mass. These fits for $\mathrm{C}$ and $\mathrm{Fe}-\mathrm{Ti}$ are shown respectively in figures 2 and 3.

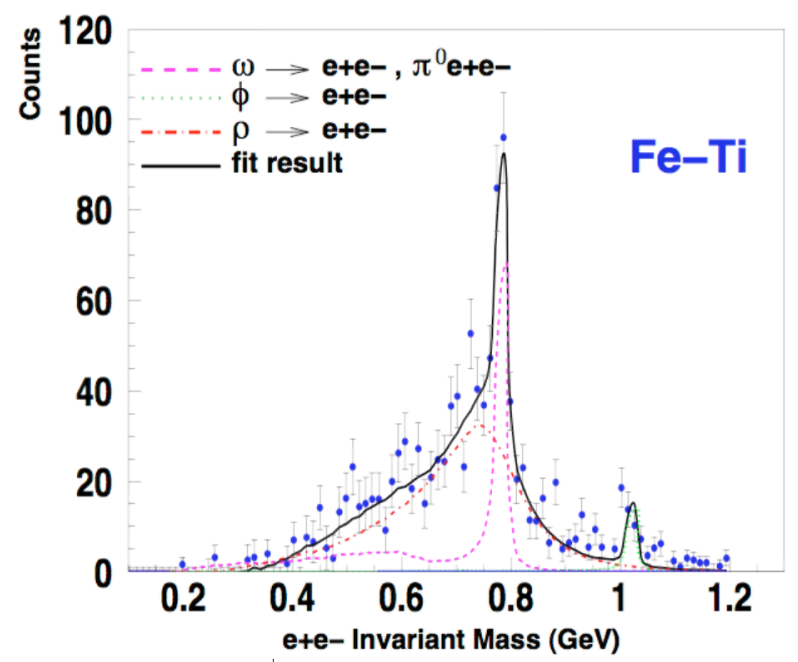

FIGURE 3. Results of the fit to the $\mathrm{e}^{+} \mathrm{e}^{-}$invariant mass obtained for Fe-Ti data. Curves are MonteCarlo calculations by the BUU model ${ }^{18,19}$ for various $\mathrm{e}^{+} \mathrm{e}^{-}$channels.

The extracted $\rho$ mass distributions and the ratio to the deuterium data are then simultaneously fit with the suggested functional form of $1 / \mathrm{m}^{3}$ times a Breit-Wigner function. ${ }^{[20-22]}$

The results of the fits are shown in figures 4,56 and tabulate in Table 1. The fits describe the data very well. The masses are consistent with the PDG values and the widths are consistent with the collisional broadening. We don't observe the doubling of the $\rho$ width reported by NA60. ${ }^{23,24}$ Our results do not favor the prediction of Brown and Rho for the mass shift (20\%) or Hatsuda and Lee ( $\alpha=0.16 \pm 0.06)$. Fit results on carbon data indicate no sensitivity to the mass shift predicted by the Hatsuda and Lee model, while Fe data do not favor the predicted shift.

\begin{tabular}{|c|c|c|c|c|}
\hline Target & $\begin{array}{c}\text { Mass }(\mathrm{MeV}) \\
\text { data }\end{array}$ & $\begin{array}{c}\text { Width (MeV) g7 } \\
\text { data }\end{array}$ & Mass (MeV) BUU & Width (MeV) BUU \\
\hline $\mathrm{D}_{2}$ & $770.3 \pm 3.2$ & $185.2 \pm 8.6$ & No BUU & No BUU \\
\hline $\mathrm{C}$ & $768.5 \pm 3.7$ & $176.4 \pm 9.5$ & $773.8 \pm 0.9$ & $177.6 \pm 2.1$ \\
\hline $\mathrm{Fe}$ & $779.0 \pm 5.7$ & $217.7 \pm 14.5$ & $773.8 \pm 5.3$ & $202.5 \pm 11.6$ \\
\hline
\end{tabular}




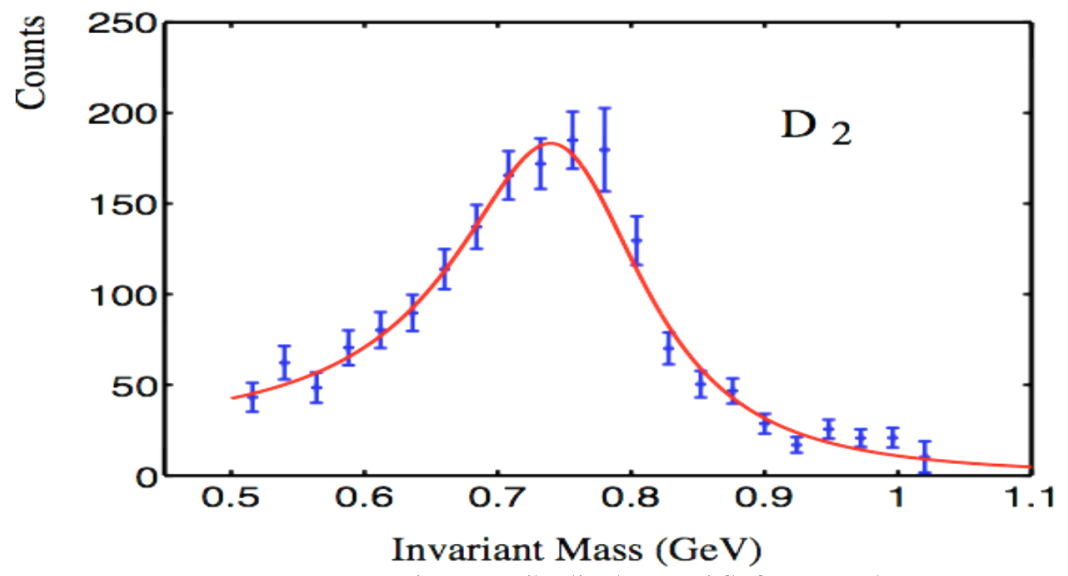

FIGURE 4. Extracted $\rho$ mass distributions and fit for Deuterium.

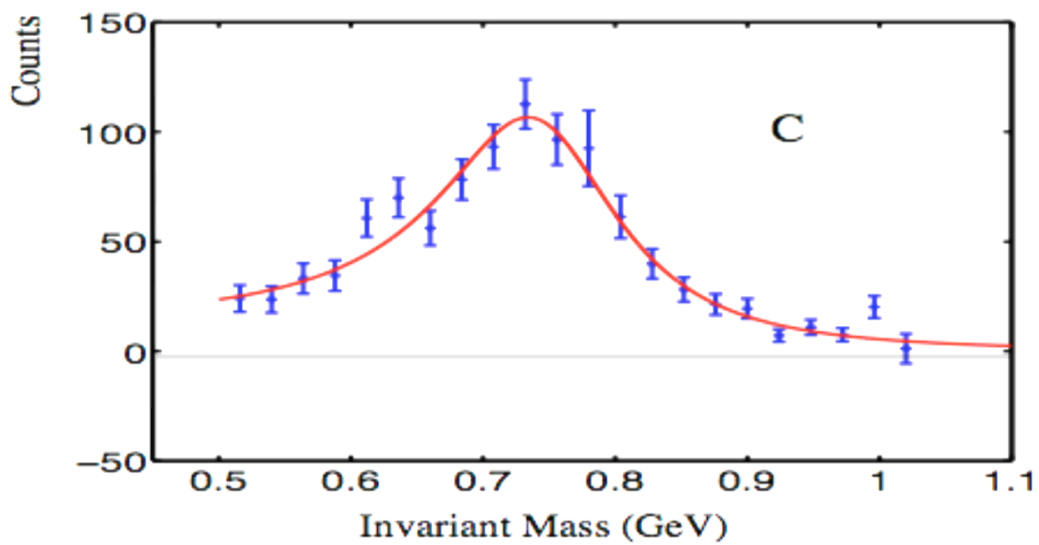

FIGURE 5. Extracted $\rho$ mass distributions and fit for Carbon.

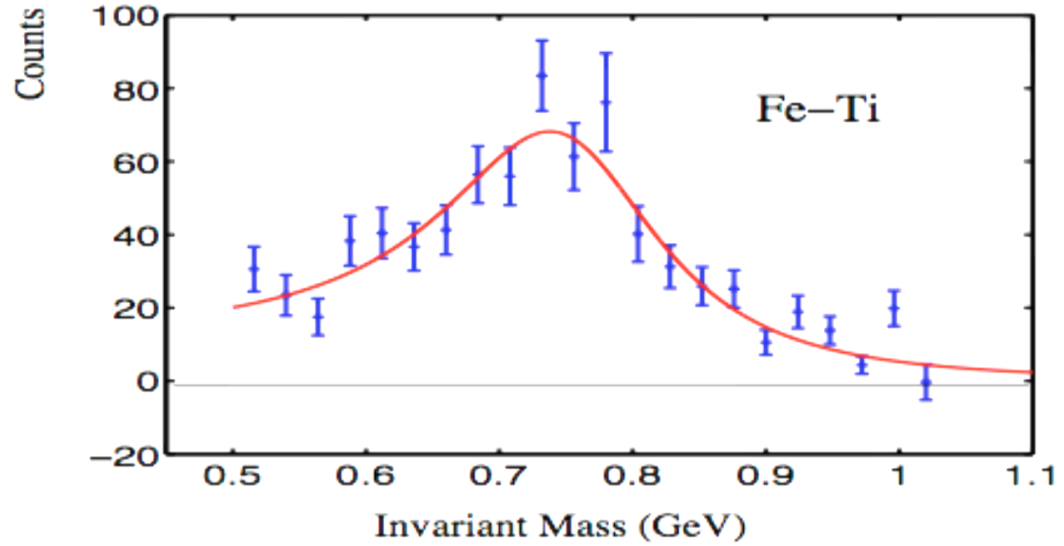

FIGURE 6. Extracted $\rho$ mass distributions and fit for Iron-Titanium. 


\section{ACKNOWLEDGMENTS}

The authors would like to thank U. Mosel, P. Muehlich, J. Weil, O. Buss, and A. Afanasev for providing us with theoretical support during this work. We would like to thank the staff of the Accelerator and Physics Divisions at Jefferson Laboratory who made this experiment possible. This work was supported in part by the U.S. Department of Energy and National Science Foundation, the Research Corporation, the Deutsche Forschungsgemeinschaft, the Italian Instituto Nazionale de Fisica Nucleare, the French Centre National de la Recherche Scientifique and Commissariat à l'Energie Atomique, the Korea Research Foundation, the U.K. Engineering and Physical Science Research Council. Jefferson Science Associates (JSA) operates the Thomas Jefferson National Accelerator Facility for the United States Department of Energy under contract DE-AC05-06OR23177.

\section{REFERENCES}

1. V Bernard and U G Meissner, Nucl. Phys. A489, Issue 4, 647 (1988).

2. S Klimt et al., Phys. Lett. B249, 386 (1990).

3. G E Brown and M Rho, et al., Phys. Rev Lett. 66, 2720 (1991).

4. T Hatsuda and S Lee, Phys. Rev. C46, R34 (1992).

5. M Herrman et al., Nucl. Phys. A545, 267c (1992).

6. R Rapp et al., Nucl. Phys. A617, 472 (1997).

7. G Agakichiev et al., Phys. Rev. Lett. 75, 1272 (1995).

8. M Massera et al., Nucl. Phys. A590, 93c (1995).

9. G Q Li et al., Phys. Rev. Lett. 75, 4007 (1995).

10. M Naruki et al., Phys. Rev. Lett. 96, 092301 (2006).

11. D Trnka et al., Phys. Rev. Lett. 94, 192303, (2005).

12. C W Leemann, D R Douglas, and G A Krafft, Annu. Rev. Nucl. Part. Sci. 51, 413 (2001)

13. B A Mecking et al., Nucl. Instr. Methods A503, 513 (2003).

14. G Jancso et al., Nucl. Phys. B124, 1 (1977)

15. B D Jouan et al., IPNO-DR-02.015 (2002).

16. P Muehlich, T Falter, C Greiner, J Lehr, M Post and U Mosel, arXiv:nucl-th/0210079 (2002).

17. M Effenberger, E L Bratkovskaya and U. Mosel, Phys. Rev. C60, 044614 (1999).

18. M Effenberger and U Mosel, Phys. Rev. C62, 014605 (2000).

19. M Effenberger, E L Bratkovskaya, W. Cassing, and U. Mosel, Phys. Rev. C60, 027601 (1999).

20. Guo-Qiang Li et al., arXiv:nucl-th/9611037 v1 Nov. (1996).

21. M Effenberger et al., arXiv:nucl-th/9903026 v2 Aug. (1999).

22. H B O'Connell et al., Prog. Part. Nucl. Phys. 39, 201 (1997)

23. S Damjanovic et al., for the NA60 collaboration, Quark Matter (2005).

24. R Arnaldi et al., Phys. Rev. Lett. 96, 162302 (2006). 